\title{
Escherichia coliy Klebsiella pneumoniae comunitarias y hospitalarias productoras de $\beta$-lactamasas en hospitales de Hermosillo, Sonora
}

\author{
Moisés Navarro-Navarro, M en C,(I) Ramón Enrique Robles-Zepeda, D en C,(I)
}

Adriana Garibay-Escobar, D en C,(I) Eduardo Ruiz-Bustos, D en C.(I)

\begin{abstract}
Navarro-Navarro M, Robles-Zepeda RE, Garibay-Escobar A, Ruiz-Bustos E. Escherichia coli y Klebsiella pneumoniae comunitarias y hospitalarias productoras de $\beta$-lactamasas en hospitales de Hermosillo, Sonora

Salud Publica Mex 20I I;53:34I-344.
\end{abstract}

\section{Resumen}

Objetivo. Determinar la prevalencia de Escherichia coli y Klebsiella pneumoniae productoras de $\beta$-lactamasas de espectro extendido (BLEE) en hospitales de Hermosillo,Sonora, México. Material y métodos. Se analizaron I 4I 2 aislamientos obtenidos durante un año (2008-2009). La detección de productores de BLEE se realizó por el método de sinergia de doble disco con y sin ácido clavulánico. Resultados. Se aislaron E. coli y K. pneumoniae productores de BLEE hospitalarios (31.8 y $35.3 \%$ ) con mayor prevalencia que los comunitarios $(14.4$ y $0.0 \%)(p<0.005)$. Conclusiones. Nuestro estudio demuestra la presencia de microorganismos productores de BLEE en los tres hospitales.

Palabras clave: Escherichia coli; Klebsiella pneumoniae; betalactamasa; México
Navarro-Navarro M, Robles-Zepeda RE,

Garibay-Escobar A, Ruiz-Bustos E.

Hospital and community-acquired $\beta$-lactamases-producing

Escherichia coli and Klebsiella pneumoniae at hospitals

in Hermosillo, Sonora.

Salud Publica Mex 20I I;53:34I-344.

\section{Abstract}

Objective. To determine the prevalence of extendedspectrum $\beta$-lactamases (ESBL)-producing Esherichia coli and Klebsiella pneumoniae in hospitals of Hermosillo, Sonora, Mexico. Material and Methods. To detect ESBL-production, I 412 bacterial isolates obtained over a one year period (2008-2009) were analyzed using the double-disk synergy test, with and without clavulanic acid. Results. Hospitalaryacquired ESBL-producing E. coli and K. pneumoniae (3I.8\% and $35.3 \%$ ) were isolated with higher prevalence that communityacquired isolates $(14.4 \%$ and $0.0 \%)(p<0.005)$. Conclusions. Our study shows the presence of ESBL-producing bacteria in the three hospitals.

Key words: Escherichia coli; Klebsiella pneumoniae; betalactamase; Mexico
$\mathrm{L}$ as beta-lactamasas de espectro extendido (BLEE) inactivan a las cefalosporinas de tercera generación y a los monobactámicos. ${ }^{1}$ Los microorganismos productores de BLEE frecuentemente son multirresistentes a distintos antimicrobianos, con excepción de los carbapenémicos y cefamicinas. ${ }^{2}$ La producción de BLEE se relaciona con fallas terapéuticas y es un serio problema para el control de infecciones en los hospitales. ${ }^{3}$ Escherichia coli y Klebsiella pneumoniae se encuentran entre los microorganismos productores de BLEE y son causa frecuente de infecciones hospitalarias y comunitarias. ${ }^{4,5}$ Es importante estudiar su epidemiología ya que repre-

(I) Departamento de Ciencias Químico Biológicas, Universidad de Sonora. Hermosillo, Sonora, México.

Recibido: septiembre 2010 - Aceptado: agosto 20II

Autor de correspondencia: M en C Moisés Navarro Navarro. Departamento de Ciencias Químico Biológicas, Universidad de Sonora. Rosales y Luis Encinas s/n, Col. Centro. 83000 Hermosillo, Sonora, México.

Correo electrónico: moisesn@guayacan.uson.mx 
sentan un problema creciente en las instituciones de salud. ${ }^{6}$ En el presente estudio, se evalúa la prevalencia de Escherichia coli y Klebsiella pneumoniae productores de BLEE y sus características microbiológicas en hospitales de Hermosillo, Sonora, México.

\section{Material y métodos}

Se realizó un estudio prospectivo del 1 de septiembre de 2008 al 31 de agosto de 2009. Se identificaron 1412 aislamientos como Escherichia coli o Klebsiella pneumoniae en los laboratorios clínicos del Centro Médico Dr. Ignacio Chávez del Instituto de Seguridad y Servicios Sociales de los Trabajadores del Estado de Sonora (CMIC), Hospital Infantil del Estado de Sonora (HIES) y Hospital San José de Hermosillo (HSJH). El proyecto de investigación, sus objetivos y metodología fueron aprobados por una comisión científica de la Universidad de Sonora. Se incluyeron los aislamientos causantes de infección comunitaria, nosocomial o colonizadores y se registró la muestra clínica para cada aislamiento. Las pruebas de identificación y susceptibilidad se realizaron utilizando los sistemas Vitek2 y MicroScan. Para la evaluación fenotípica de producción de BLEE se empleó el método de sinergia de doble disco con y sin ácido clavulánico. ${ }^{7}$ Se utilizaron las cepas control Escherichia coli 25922 y Klebsiella pneumoniae
700603. Se determinó la ji cuadrada o la prueba exacta de Fisher utilizando el paquete estadístico NCSS. Se consideró significativo el valor de $p<0.05$.

\section{Resultados}

Se estudiaron 1412 aislamientos de los cuales 1184 (83.9\%) fueron E. coli y 228(16.1\%) K. pneumoniae (cuadro I); de los anteriores, 239 (44.7\%) y $102(20.2 \%)$ correspondieron con aislamientos hospitalarios respectivamente. En el porcentaje acumulado se observó una mayor prevalencia de E. coli y K. pneumoniae productores de BLEE hospitalarios (31.8 y $35.3 \%$ ) que comunitarios (14.4 y $0.0 \%)(p<0.005)$ (cuadro I), con excepción de los aislamientos de E. coli en el HSJH (cuadro I). En el cuadro II se observa que los productores de BLEE fueron aislados con mayor prevalencia de infecciones urinarias comunitarias $(0.0-64.9 \%)$ y hospitalarias $(0.0-50.0 \%)$, seguido de sangre $(0.0-26.5 \%)$, piel y tejido blando $(8.2-17.2 \%)$, éstas últimas de origen hospitalario. Los productores de BLEE fueron comúnmente sensibles a los carbapenémicos (94.0-100\%) y amikacina (45.0-62.0\%). Para $E$. coli resalta la sensibilidad a nitrofurantoína $(83.0 \%)$ en el CMIC e HIES (86.0\%) y para K. pneumoniae (HIES) la sensibilidad a quinolonas (91.0-94.0\%) y trimetoprim/ sulfametoxazol $(91.0 \%)$.

Cuadro I

Distribución de los microorganismos PRODUCTORES DE BLEE EN LOS HOSPITALES PARTICIPANTES. Hermosillo, Sonora, México, 2008-2009

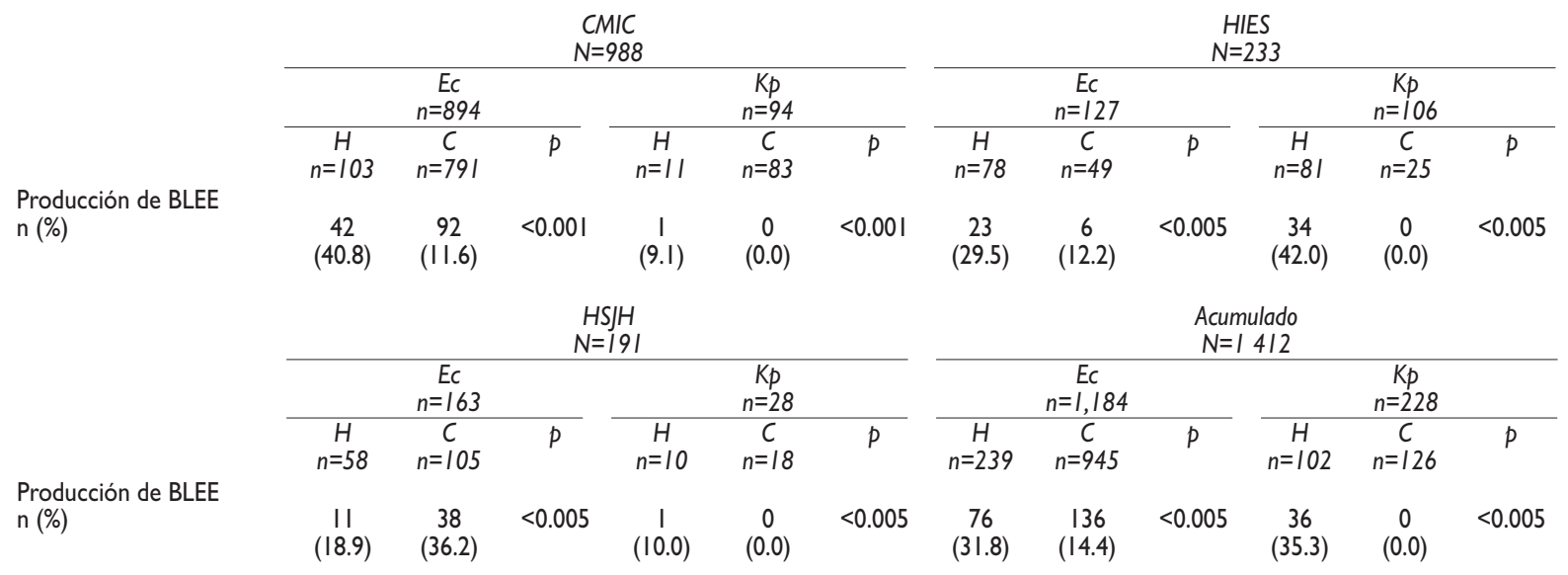

CMIC: Centro Médico Dr. Ignacio Chávez del ISSSTESON

HIES: Hospital Infantil del Estado de Sonora

HSJH: Hospital San José de Hermosillo

Ec: Escherichia coli

Kp: Klebsiella pneumoniae

$\mathrm{H}$ : Hospitalario

C: Comunitario

BLEE: $\beta$-lactamasas de espectro extendido 


\section{Cuadro II \\ Distribución de los Aislamientos PROdUCTORES DE BLEE POR ORIGEN Y SITIO DE INFECCIÓN. Hermosillo, Sonora, México, 2008-2009}

\begin{tabular}{|c|c|c|c|c|c|c|}
\hline \multirow[b]{2}{*}{$\begin{array}{l}\text { Origen del } \\
\text { aislamiento }\end{array}$} & \multirow{2}{*}{ Tejido o tracto } & \multirow{2}{*}{$\begin{array}{c}\text { CMIC } \\
E C \\
n=134\end{array}$} & \multicolumn{2}{|c|}{ HIES } & \multirow{2}{*}{$\begin{array}{c}H S J H \\
E C \\
n=49\end{array}$} & \multirow[b]{2}{*}{$p$} \\
\hline & & & $\begin{array}{c}E c \\
n=29\end{array}$ & $\underset{n=34}{K p}$ & & \\
\hline \multirow{4}{*}{$\begin{array}{l}\text { Hospitalario } \\
\mathrm{n}(\%)\end{array}$} & Vías urinarias & $16(11.9)$ & $13(44.8)$ & $17(50.0)$ & $0(0.0)$ & $<0.005$ \\
\hline & Piel y tejido blando & $20(14.9)$ & $5(I 7.2)$ & $3(8.8)$ & $4(8.2)$ & 0.48 \\
\hline & Sangre & $4(3.0)$ & $5(17.2)$ & $9(26.5)$ & $0(0.0)$ & 0.001 \\
\hline & TG,TGI y Otros* & $2(1.5)$ & $0(0.0)$ & $5(14.7)$ & $7(14.3)$ & $<0.001$ \\
\hline \multirow{2}{*}{$\begin{array}{l}\text { Comunitario } \\
\mathrm{n}(\%)\end{array}$} & Vías urinarias & 87 (64.9) & $3(10.3)$ & $0(0.0)$ & $38(77.6)$ & $<0.001$ \\
\hline & TG,TGI & $5(3.7)$ & $3(10.3)$ & $0(0.0)$ & $0(0.0)$ & 0.056 \\
\hline
\end{tabular}

*Se incluyen sistema nervioso central, tracto respiratorio superior e inferior y líquidos diversos.

CMIC: Centro Médico Dr. Ignacio Chávez del ISSSTESON

HIES: Hospital Infantil del Estado de Sonora

HSJH:Hospital San José de Hermosillo

Ec: Escherichia coli

Kp: Klebsiella pneumoniae

TG:Tracto genital

TGI:Tracto gastrointestinal

\section{Discusión}

El sistema de vigilancia SENTRY informa una prevalencia de 45.0 y $8.5 \%$ en América Latina, 7.6 y $3.3 \%$ en Estados Unidos y 22.6 y $5.3 \%$ en Europa para E. coli y K. pneumoniae productoras de BLEE respectivamente. ${ }^{8}$ Se detectó un incremento de $18.4 \%$ en la prevalencia total de E. coli y de $25.4 \%$ para K. pneumoniae productoras de BLEE en el HIES, en comparación con un estudio de prevalencia realizado de 2002 a 2003 (4.4 y 6.7\% respectivamente). ${ }^{9}$ Silva-Sánchez y col. ${ }^{10}$ informaron que K. pneumoniae es la enterobacteria productora de BLEE de origen nosocomial con mayor frecuencia de aislamientos (56.0\%) en hospitales mexicanos, seguida de Enterobacter cloacae (29.0\%) y Escherichia coli (15.0\%). En Europa, los productores de BLEE son causantes frecuentes de infecciones en sangre, tracto urinario, tracto respiratorio, piel y tejido blando. ${ }^{11}$ En Canadá, Mulvey y col. ${ }^{12}$ informaron que la infección urinaria hospitalaria es donde frecuentemente se aíslan $E$. coli y K. pneumoniae productores de BLEE. Los microorganismos productores de BLEE son causantes de infección en pacientes con factores de riesgo y hospitalizados..$^{13}$ Son cada vez más los informes del hallazgo comunitario de E. coli uropatógena productora de BLEE. ${ }^{2}$ Coque y col., ${ }^{11}$ en Turquía, observaron una prevalencia de $21.0 \%$ en el aislamiento de E. coli productora de BLEE uropatógena comunitaria. En el presente estudio se detectaron E. coli y K. pneumoniae productoras de BLEE como causantes de diversas infecciones hospitalarias y comunitarias, regularmente sensibles a los carbapenémicos. Los informes científicos confirman que los carbapenémicos son los antibióticos de primera elección para el tratamiento de las infecciones graves causadas por microorganismos productores de BLEE. ${ }^{3}$ La vigilancia constante de la prevalencia de microorganismos productores de BLEE en los hospitales contribuye a conocer la dimensión del problema y a definir estrategias para su control.

Declaración de conflicto de intereses: Los autores declararon no tener conflicto de intereses.

\section{Referencias}

I. Katz OT, Peled N, Yagupsky P. Evaluation of the current National Committee for Clinical Laboratory Standards guidelines for screening and confirming extended-spectrum beta-lactamase production in isolates of Escherichia coli and Klebsiella species from bacteremic patients. Eur J Clin Microbiol Infect Dis 2004;23:813-817.

2. Hernández JR, Pascual A, Cantón R, Martínez-Martínez L. Grupo de estudio de infección hospitalaria (GEIH). Escherichia coli y Klebsiella pneumoniae productores de B-lactamasas de espectro extendido en hospitales españoles (Proyecto GEIH-BLEE 2000). Enferm Infecc Microbiol Clin 2003;21:77-82.

3. Pitout JD, Laupland KB. Extended-spectrum beta-lactamase-producing Enterobacteriaceae: an emerging public-health concern. Lancet Infect Dis 2008;8:159-166. 
4. Falagas ME, Karageorgopoulos DE. Extended-spectrum $\beta$-lactamaseproducing organisms. J Hosp Inf 2009;73:345-354.

5. Silva J, Gatica R, Aguilar C, Becerra Z, Garza-Ramos U,Velázquez

$M$, et al. Outbreak of infection with extended-spectrum $\beta$-lactamaseproducing Klebsiella pneumoniae in a mexican hospital. J Clin Microbiol 2001;39:3193-3196.

6. Garza-Ramos U, Martínez-Romero E, Silva-Sánchez J. SHV-type extended-spectrum $\beta$-lactamase (ESBL) are encoded in related plasmids from enterobacteria clinical isolates from Mexico. Salud Publica Mex 2007:49:415-42I.

7. CLSI. Performance standards for antimicrobial susceptibility testing; eighteenth informational supplement. Clinical and Laboratory Standards Institute 2008. MI00-SI8. Wayne, PA.

8. Winokur PL, Canton R, Casellas JM, Legakis N.Variations in the prevalence of strains expressing an extended-spectrum $\beta$-lactamase phenotype and characterization of isolates from Europe, the Americas, and the Western Pacific Region. Clin Inf Dis 200 I;32(Suppl 2):S94- 103.

9. Navarro-Navarro M, Moreno-Noriega BO, López-Munguía BE, FragosoCarmelo MC, Sánchez-Padilla JA. Detección de cepas de Escherichia coli y
Klebsiella pneumoniae productoras de $\beta$-lactamasas de espectro extendido (BLEE) en el Hospital Infantil del Estado de Sonora. Bol Clin Hosp Inf Edo Son 2005;22:64-70.

10. Silva-Sanchez J, Garza-Ramos JU, Reyna-Flores F, Sanchez-Perez $\mathrm{A}$, Rojas-Moreno T, Andrade-Almaraz $\mathrm{V}$, et al. Extended-spectrum $\beta$-lactamase-producing Enterobacteriaceae causing nosocomial infections in Mexico.A retrospective and multicenter study. Arch Med Res 201I;42:156-162.

II. Coque TM, Baquero F, Canton R. Increasing prevalence of ESBLproducing enterobacteriaceae in Europe. Eurosurveillance 2008; I3: I- II. 12. Mulvey MR, Bryce E, Boyd D, Ofner-Agostini M, Christianson S, Simor $\mathrm{AE}$, et al.Ambler class $\mathrm{A}$ extended-spectrum beta-lactamase-producing Escherichia coli and Klebsiella spp. in Canadian hospitals. Antimicrobial Agents Chemother 2004;48: I204-I2I4.

13. Gniadkowsky M. Evolution and epidemiology of extended-spectrum $\beta$-lactamases (ESBLs) and ESBL-producing microorganisms. Clin Microbiol Infect 200I;7:597-608. 